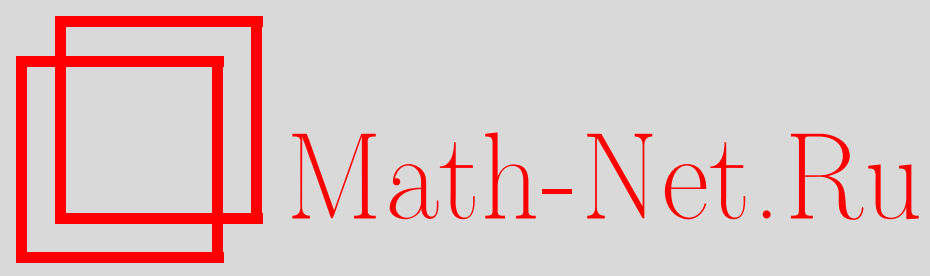

Н. Н. Воробьев, А. Н. Скиба, О дистрибутивности решетки разрешимых тотально локальных классов Фиттинга, $M a$ тем. заметки, 2000, том 67, выпуск 5, 662-673

DOI: https://doi.org/10.4213/mzm882

Использование Общероссийского математического портала Math-Net.Ru подразумевает, что вы прочитали и согласны с пользовательским соглашением http://www.mathnet.ru/rus/agreement

Параметры загрузки:

IP : 54.224 .135 .184

26 апреля 2023 г., $16: 36: 40$

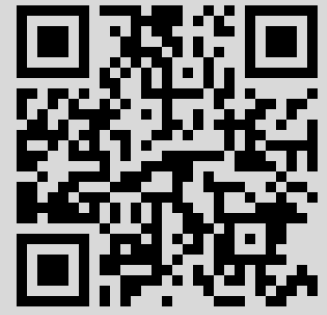




\title{
О ДИСТРИБУТИВНОСТИ РЕШЕТКИ РАЗРЕШИМЫХ ТОТАЛЬНО ЛОКАЛЬНЫХ КЛАССОВ ФИТТИНГА
}

\author{
Н. Н. Воробъёв, А. Н. Скиба
}

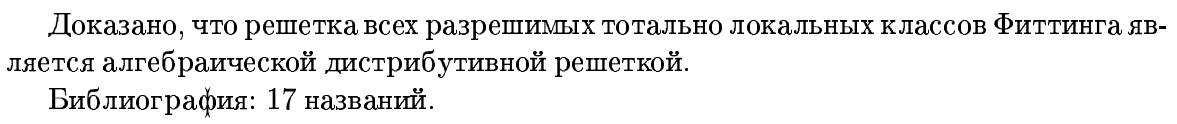

Все рассматриваемые групшы конечны. Напомним, что функции вида $f: \mathbb{P} \mapsto\{$ формации $\}$ назьваются формационными функииями [1] или, иначе, спутниками [2]. Если класс групп $\mathfrak{F}$ таков, что

$$
\mathfrak{F}=\mathfrak{G}_{\pi(\mathfrak{F})} \cap\left(\bigcap_{p \in \pi(\mathfrak{F})} \mathfrak{G}_{p^{\prime}} \mathfrak{N}_{p} f(p)\right)
$$

где $f$ - некоторьй спутник, то говорят, что $\mathfrak{F}$ - локальная формация со спутником $f$ и пишут $\mathfrak{F}=\mathrm{LF}(f)$. Здесь $\pi(\mathfrak{F})$ - множество всех простых делителей порядков групп из $\mathfrak{F}$, символы $\mathfrak{N}_{p}$ и $\mathfrak{G}_{p^{\prime}}$ обозначают класс всех $p$-групा и класс всех $p^{\prime}$-групп соответственно.

$\Phi$ ункции вида $f: \mathbb{P} \mapsto\{$ классы Фиттинга $\}$ называются функииями Хартли или, более коротко, $H$-функииями [2]. Если для класса групп $\mathfrak{F}$ имеет место

$$
\mathfrak{F}=\mathfrak{G}_{\pi(\mathfrak{F})} \cap\left(\bigcap_{p \in \pi(\mathfrak{F})} f(p) \mathfrak{N}_{p} \mathfrak{G}_{p^{\prime}}\right)
$$

где $f$ - некоторая $H$-функция, то говорят, что $\mathfrak{F}$ - локальный класс Фиттинга с $H$-функцией $f$ и пишут $\mathfrak{F}=\operatorname{LR}(f)$.

При обозрении большинства наиболее известных конкретных классов групп легко обнаруживается, что они могут быть заданы при помощи функций, все непустые значения которых сами являются локальными классами. Последнее обстоятельство привело к возникновению следующей естественной конструкции [3]: всякая формация считается 0 -кратно локальной; а при $n \geqslant 1$ формация $\mathfrak{F}$ назьвается $n$-кратно локальной, если $\mathfrak{F}=\mathrm{LF}(f)$, где все непустые значения спутника $f(n-1)$-кратно локальны. Формация назьвается тотально локальной, если она $n$-кратно локальна для всех натуральных $n$. Аналогично определяются $n$-кратно локальные и тотально локальные классы Фиттинга. Нетрудно показать, что класс разрешимых тотально локальных формаций совпадает с классом так называемых примитивных насьшенных формаций, введенньм Хоуксом 
в работе [4]. Кратно локальные классы нашли приложения при решении многих вопросов теории классов (см., например, [5]-[10]).

Являясь предельным случаем $n$-кратно локальных классов, тотально локальныеклассы обладают рядом специфических свойств. Отметим, в частности, что при любом целом неотрицательном $n$ решетки всех $n$-кратно локальных формаций, $n$-кратно локальных наследственных формаций, $n$-кратно локальных нормально наследственных формаций и т.д. являются модулярными, но все они не дистрибутивны даже в классе разрешимых групп $\mathfrak{S}$ (см. [5, гл. 2] и [6, гл. 4]). Что же касается тотально локальных формаций, то в настоящее время неизвестно, является ли хотя бы модулярной решетка всех тотально локальных $\tau$-замкнутых формаций хотя бы для одного нетривиального подгруппового функтора $\tau$ [6, вопрос 4.2.14]. В то же время в монографии [5] анонсирован результат о том, что решетка всех разрешимых тотально локальных формаций дистрибутивна. В данной работе мы доказьваем, что решетка всех разрешимых тотально локальных классов Фиттинга является алгебраической и дистрибутивной и такой, в которой любой элемент, отличньй от (1) и $\mathfrak{S}$, не дополняем. В ходеполучения такого результата мы выявляем ряд общих свойств оператора порождения $\vee^{\infty}$. C другой стороны, в качестве одного из следствий мы даем здесь полное доказательство дистрибутивности решетки всех разрешимых тотально локальных формаций, другая схема которого обсуждалась в монографии [6].

Напомним некоторые определения [2], связанные с локальными классами Фиттинга. Символом $F^{p}(G)$ обозначают подгруппу $O^{p}\left(O^{p^{\prime}}(G)\right)$. Символом $l^{\infty}$ обозначается решетка всех тотально локальных классов Фиттинга. $H$-Функция $f$ называется $l^{\infty}$-значной [6], если каждое ее непустое значение принадлежит решетке $l^{\infty}$. Пусть $\left\{f_{i}(p) \mid\right.$ $i \in I\}$ - произвольньй набор $l^{\infty}$-значных $H$-функций. Для всякого $p \in \mathbb{P}$ положим $\left(\bigcap_{i \in I} f_{i}\right)(p)=\bigcap_{i \in I} f_{i}(p) . H$-Функция $\bigcap_{i \in I} f_{i}$ называется пересечением $H$-функций $f_{i}$. Если класс Фиттинга $\mathfrak{F}$ обладает по крайней мере одной $l^{\infty}$-значной $H$-функцией, то пересечение всех таких $H$-функций класса $\mathfrak{F}$ назьвается минимальной $l^{\infty}$-значной $H$-функиией класса $\mathfrak{F}$.

Пусть $\mathfrak{X}$ - произвольная непустая совокупность групш. Пересечение всех тотально локальных классов Фиттинга, содержащих $\mathfrak{X}$, обозначают через $l^{\infty}$ fit $\mathfrak{X}$ и назьвают тотально локальным классом Фиттинга, порожденным $\mathfrak{X}[2]$. Если $\mathfrak{X}=\{G\}$, то вместо $l^{\infty}$ fit $\{G\}$ пишут $l^{\infty}$ fit $G$. Всякий класс $\Phi$ иттинга такого вида называется однопорожденным тотально локальным классом Фиттинга [2].

Нам потребуется следующий частньй случай леммы 21 работы [2].

ЛЕмма 1. Если $\mathfrak{F}=l^{\infty}$ fit $\mathfrak{X} u f-$ минимальная $l^{\infty}$-значная $H$-функиия класса $\mathfrak{F}$, mo

$$
f(p)=l^{\infty} \operatorname{fit}\left(F^{p}(G) \mid G \in \mathfrak{X}\right)
$$

при всех $p \in \pi(\mathfrak{X})$ u $f(p)=\varnothing$ при всех $p \in \mathbb{P} \backslash \pi(\mathfrak{X})$.

ДокАЗАтЕЛьство. Пусть $t-H$-функция такая, что $t(p)=l^{\infty} \operatorname{fit}\left(F^{p}(G) \mid G \in \mathfrak{X}\right)$ при всех $p \in \pi(\mathfrak{X})$ и $t(p)=\varnothing$ при всех $p \in \mathbb{P} \backslash \pi(\mathfrak{X})$. Покажем, что $t=f$. Пусть $\mathfrak{M}=\operatorname{LR}(t)$. Тогда, очевидно, $\mathfrak{X} \subseteq \mathfrak{M}$. Значит, $\mathfrak{F} \subseteq \mathfrak{M}$. Пусть $f_{1}-$ произвольная $l^{\infty}$-значная $H$-функция класса $\mathfrak{F}$. Тогда поскольку $\mathfrak{X} \subseteq \mathfrak{F}$, для любого $p \in \mathbb{P}$ имеет место $\left(F^{p}(G) \mid G \in \mathfrak{X}\right) \subseteq f_{1}(p)$. Значит, $t(p) \subseteq f_{1}(p)$. Поэтому $\mathfrak{M} \subseteq \mathfrak{F}$. Следовательно, $\mathfrak{M}=\mathfrak{F}$ и $t=f$. Лемма доказана. 
Пусть $\left\{\mathfrak{F}_{i} \mid i \in I\right\}$ - произвольньй набор классов Фиттинга из $l^{\infty}$. Тогда через $\vee^{\infty}\left(\mathfrak{F}_{i} \mid i \in I\right)$ обозначается [6] верхняя грань для $\left\{\mathfrak{F}_{i} \mid i \in I\right\}_{\text {в }} l^{\infty}$, а через $\vee^{\infty}\left(f_{i} \mid i \in I\right)$ - $H$-функция $f$ такая, что $f(p)$ является верхней гранью для $\left\{f_{i}(p) \mid i \in I\right\}$ в $l^{\infty}$, если $\bigcup_{i \in I} f_{i}(p) \neq \varnothing$, и $f(p)=\varnothing$ в противном случае.

Лемма 2. Пусть $f_{i}-$ минимальная $l^{\infty}$-значная $H$-функиия класса Фиттинга $\mathfrak{F}_{i}$, $i \in I$. Тогда $\vee^{\infty}\left(f_{i} \mid i \in I\right)-$ минимальная $l^{\infty}$-значная $H$-функиия класса Фиттинга $\mathfrak{F}=\vee^{\infty}\left(\mathfrak{F}_{i} \mid i \in I\right)$.

ДОКАЗАТЕЛЬСТво. Пусть $\pi=\pi\left(\bigcup_{i \in I} \mathfrak{F}_{i}\right)=\bigcup_{i \in I} \pi\left(\mathfrak{F}_{i}\right)=\pi(\mathfrak{F}), f=\vee^{\infty}\left(f_{i} \mid i \in I\right)$ и $h$ - минимальная $l^{\infty}$-значная $H$-функция класса Фиттинга $\mathfrak{F}$. Покажем, что $h=f$.

Пусть $p \in \mathbb{P} \backslash \pi$. Тогда для любого $i \in I$ имеет место $h(p)=\varnothing$ и $f_{i}(p)=\varnothing$. Значит, $f(p)=\varnothing$.

Пусть $p \in \pi$. Тогда найдется $i \in I$ такое, что $f_{i}(p) \neq \varnothing$. По лемме 1 имеет место

$$
\begin{aligned}
h(p) & =l^{\infty} \operatorname{fit}\left(\left(F^{p}(G) \mid G \in \bigcup_{i \in I} \mathfrak{F}_{i}\right)\right)=l^{\infty} \text { fit }\left(\left(\bigcup_{i \in I} l^{\infty} \operatorname{fit}\left(\left(F^{p}(G) \mid G \in \mathfrak{F}_{i}\right)\right)\right)\right) \\
& =l^{\infty} \operatorname{fit}\left(\left(\bigcup_{i \in I} f_{i}(p)\right)\right)=\left(\left(\vee^{\infty}\left(f_{i} \mid i \in I\right)\right)\right)(p)=f(p) .
\end{aligned}
$$

Лемма доказана.

$H$-Функция $f$ называется внутренней, если $f(p) \subseteq \operatorname{LR}(f)$ для всех $p \in \mathbb{P}$. В дальнейшем нам будет необходима следующая лемма, являющаяся частным случаем результата, полученного Н. Т. Воробьевым [11].

ЛЕмма 3. Для любого тотально локального класса Фиттинга $\mathfrak{H}$ класс $\mathfrak{H N}_{p}$ тотально локален.

Лемма $4\left[2\right.$, лемма 23]. Пусть $\mathfrak{F}=\operatorname{LR}(f)$. Тогда если $O^{p}(G) \in f(p) \cap \mathfrak{F}$, mo $G \in \mathfrak{F}$.

Со всяким классом Фиттинга можно сопоставить наименьший (по включению) класс Фиттинга $\mathfrak{F}^{*}[12]$, содержащий $\mathfrak{F}$ и такой, что для любых групा $G$ и $H$ справедливо равенство $(G \times H)_{\mathfrak{F}^{*}}=G_{\mathfrak{F}^{*}} \times H_{\mathfrak{F}^{*}}$. Класс Фиттинга $\mathfrak{F}$ называется классом Локетта, если $\mathfrak{F}=\mathfrak{F}^{*}$.

Лемма 5. Если $\left\{\mathfrak{F}_{i}=\operatorname{LR}\left(f_{i}\right) \mid i \in I\right\}-$ набор тотально локальных классов Фиттинга, где $f_{i}$ - некоторая внутренняя $l^{\infty}$-значная $H$-функиия, то

$$
\vee^{\infty}\left(\mathfrak{F}_{i} \mid i \in I\right)=\operatorname{LR}\left(\vee^{\infty}\left(f_{i} \mid i \in I\right)\right)
$$

ДокаЗАТЕЛЬСтво. Пусть $\mathfrak{F}=\vee^{\infty}\left(\mathfrak{F}_{i} \mid i \in I\right), \mathfrak{M}=\operatorname{LR}\left(\vee^{\infty}\left(f_{i} \mid i \in I\right)\right)$ и $h_{i}-$ минимальная $l^{\infty}$-значная $H$-функция класса $\mathfrak{F}_{i}$. Тогда по лемме $2 h=\mathrm{V}^{\infty}\left(h_{i} \mid i \in I\right)-$ минимальная $l^{\infty}$-значная $H$-функция класса $\mathfrak{F}$. Поскольку $h_{i} \leqslant f_{i}$, для всех $p \in \mathbb{P}$ имеет место включение

$$
l^{\infty} \text { fit }\left(\bigcup_{i \in I} h_{i}(p)\right) \subseteq l^{\infty} \text { fit }\left(\bigcup_{i \in I} f_{i}(p)\right) .
$$

Отсюда $\mathfrak{F} \subseteq \mathfrak{M}$. 
Докажем теперь обратное включение. Пусть $t_{i}-H$-функция класса $\mathfrak{F}_{i}$ такая, что $t_{i}(p)=h_{i}(p) \mathfrak{N}_{p}$ для всех $p \in \mathbb{P}$. Ввиду леммы 3 эта $H$-функция $l^{\infty}$-значна. Покажем, что $f_{i} \leqslant t_{i}$.

Допустим, что $f_{i} \nless t_{i}$. Тогда найдется простое число $p$ такое, что $f_{i}(p) \nsubseteq t_{i}(p)$. Пусть $G$ - группа из $f_{i}(p) \backslash t_{i}(p)$. Пусть $\Gamma=G \succ Z_{p}=[K] Z_{p}$, где $Z_{p}$ - группа порядка $p, K$ - база регулярного сплетения $\Gamma$. Поскольку каждый локальньй класс Фиттинга является классом Локетта [13] и $G \notin t_{i}(p)$, согласно [1, гл. Х, утверждение $\left.2.1 \mathrm{a}\right)$ ] $\Gamma_{t_{i}(p)}=K_{1}$, где $K_{1}$ - база регулярного сплетения $\Gamma_{1}=G_{t_{i}(p)} \prec Z_{p}$. Ввиду свойств сплетений (см., например, [1, гл. А, $18.2 \mathrm{~d})]$ )

$$
\Gamma / \Gamma_{t_{i}(p)}=\Gamma / K_{1} \simeq\left(G / G_{t_{i}(p)}\right) \curlywedge Z_{p} .
$$

Следовательно, $p$ делит порядок $\Gamma / \Gamma_{t_{i}(p)}$.

Так как $G \in f_{i}(p)$, то $K \in f_{i}(p)$. Поэтому $K \subseteq \Gamma_{f_{i}(p)}$. Поскольку $\Gamma / K \simeq Z_{p} \in \mathfrak{N}_{p}$, то

$$
\Gamma / K / \Gamma_{f_{i}(p)} / K \simeq \Gamma / \Gamma_{f_{i}(p)} \in \mathfrak{N}_{p} .
$$

Значит, по лемме $4 \Gamma \in f_{i}(p) \mathfrak{N}_{p} \subseteq \mathfrak{F}_{i}=\operatorname{LR}\left(f_{i}\right)=\operatorname{LR}\left(t_{i}\right)$ и поэтому

$$
\Gamma \in \mathfrak{G}_{\pi(\mathfrak{F})} \cap\left(\bigcap_{q \in \pi(\mathfrak{F})} t_{i}(q) \mathfrak{G}_{q^{\prime}}\right)
$$

и, в частности, $\Gamma \in t_{i}(p) \mathfrak{G}_{p^{\prime}}$ для всех $p \in \pi(\mathfrak{F})$. Следовательно, $\Gamma / \Gamma_{t_{i}(p)} \in \mathfrak{G}_{p^{\prime}}$. Противоречие.

Итак, $f_{i} \leqslant t_{i}$. Значит, $f=\vee^{\infty}\left(f_{i} \mid i \in I\right) \leqslant \vee^{\infty}\left(t_{i} \mid i \in I\right)$, т.е. для любого $p \in \mathbb{P}$ имеет место включение

$$
f(p)=\vee^{\infty}\left(f_{i}(p) \mid i \in I\right) \subseteq \vee^{\infty}\left(t_{i}(p) \mid i \in I\right)=\vee^{\infty}\left(h_{i}(p) \mathfrak{N}_{p} \mid i \in I\right) .
$$

Поскольку $h_{i}(p) \mathfrak{N}_{p} \subseteq\left(\vee^{\infty}\left(h_{i}(p) \mid i \in I\right)\right) \mathfrak{N}_{p}$, то

$$
l^{\infty} \text { fit }\left(\bigcup_{i \in I} h_{i}(p) \mathfrak{N}_{p}\right) \subseteq l^{\infty} \text { fit }\left(\vee^{\infty}\left(h_{i}(p) \mid i \in I\right) \mathfrak{N}_{p}\right)=\left(\vee^{\infty}\left(h_{i}(p) \mid i \in I\right)\right) \mathfrak{N}_{p}
$$

для всех $p \in \mathbb{P}$. Hо $\mathfrak{F}=\operatorname{LR}(t)$, где $t-l^{\infty}$-значная $H$-функция такая, что

$$
t(p)=\left(\vee^{\infty}\left(h_{i}(p) \mid i \in I\right)\right) \mathfrak{N}_{p}
$$

для всех $p \in \mathbb{P}$. Следовательно, $f \leqslant t$. Значит, $\mathfrak{M} \subseteq \mathfrak{F}$. Поэтому $\mathfrak{M}=\mathfrak{F}$. Лемма доказана.

Лемма 6. Пусть $A \in \mathfrak{S} \cap \omega\left(\mathfrak{F}_{1}, \ldots, \mathfrak{F}_{m}\right)$, где $\omega\left(x_{1}, \ldots, x_{m}\right)-$ терм сигнатуры $\left\{\cap, \vee^{\infty}\right\}, \mathfrak{F}_{1}, \ldots, \mathfrak{F}_{m}$ - некоторые разрешимые тотально локальные классы фиттинга. Тогда найдутся группь $A_{1}, \ldots, A_{m}\left(A_{i} \in \mathfrak{F}_{i}\right)$ такие, что 
ДОКАЗАТЕЛЬСТВо. Индукцией по числу $r$ вхождений символов из $\left\{\cap, \vee^{\infty}\right\}$ в терм $\omega$ покажем, что найдутся группы $A_{i} \in \mathfrak{F}_{i}(i=1, \ldots, m)$ такие, что

$$
A \in \omega\left(l^{\infty} \text { fit } A_{1}, \ldots, l^{\infty} \text { fit } A_{m}\right)
$$

При $r=0$, очевидно, $A \in l^{\infty}$ fit $A$. Индукцией по нильпотентной длине группы $A$ докажем, что утверждение верно при $r=1$.

Пусть $A \in \mathfrak{F}_{1} \vee^{\infty} \mathfrak{F}_{2}=l^{\infty} \operatorname{fit}\left(\mathfrak{F}_{1} \cup \mathfrak{F}_{2}\right)$ и $\pi(A)=\left\{p_{1}, \ldots, p_{k}\right\}$. При $l(A)=1$ имеет место $A=P_{1} \times \cdots \times P_{k}$, где $P_{i}-$ силовская $p_{i}$-подгруппа группы $A$. Ясно, что $\pi(A) \subseteq \pi\left(\mathfrak{F}_{1}\right) \cup \pi\left(\mathfrak{F}_{2}\right)$. Пусть $p_{1}, \ldots, p_{j} \in \pi\left(\mathfrak{F}_{1}\right), p_{j+1}, \ldots, p_{k} \in \pi\left(\mathfrak{F}_{2}\right)$. Тогда $A_{1}=P_{1} \times \cdots \times P_{j} \in \mathfrak{F}_{1}, A_{2}=P_{j+1} \times \cdots \times P_{k} \in \mathfrak{F}_{2}$. Понятно, что

$$
A=A_{1} \times A_{2} \in\left(l^{\infty} \text { fit } A_{1}\right) \vee^{\infty}\left(l^{\infty} \text { fit } A_{2}\right)
$$

Пусть теперь $l(A)>1$. Допустим, что для всех разрешимых групा, нильпотентная длина которых меньше нильпотентной длины группы $A$, доказьваемое нами утверждение верно. Ввиду лемм 1 и 2 для произвольного $p_{i} \in \pi(A)$ имеет место

$$
F^{p_{i}}(A) \in f_{1}\left(p_{i}\right) \vee^{\infty} f_{2}\left(p_{i}\right)=\left(l^{\infty} \operatorname{fit}\left(F^{p_{i}}(G) \mid G \in \mathfrak{F}_{1}\right)\right) \vee^{\infty}\left(l^{\infty} \operatorname{fit}\left(F^{p_{i}}(G) \mid G \in \mathfrak{F}_{2}\right)\right),
$$

где $f_{j}$ - минимальная $l^{\infty}$-значная $H$-функция класса Фиттинга $\mathfrak{F}_{j}, j=1,2$. Поскольку $l\left(F^{p_{i}}(A)\right)<l(A)$, по индукции найдутся групшы $A_{i_{1}} \in f_{1}\left(p_{i}\right), A_{i_{2}} \in f_{2}\left(p_{i}\right)$ такие, что

$$
F^{p_{i}}(A) \in\left(l^{\infty} \text { fit } A_{i_{1}}\right) \vee^{\infty}\left(l^{\infty} \text { fit } A_{i_{2}}\right)
$$

Пусть $B_{i_{1}}=A_{i_{1}} \prec Z_{p_{i}}, B_{i_{2}}=A_{i_{2}} \prec Z_{p_{i}}$, где $Z_{p_{i}}$ - циклическая группа порядка $p_{i}$, $K$ - база регулярного сплетения $B_{i_{1}}$. Так как $A_{i_{1}} \in f_{1}\left(p_{i}\right)$, по лемме 4 имеет место $B_{i_{1}} \in f_{1}\left(p_{i}\right) \mathfrak{N}_{p_{i}} \subseteq \mathfrak{F}_{1}$. Аналогично, $B_{i_{2}} \in \mathfrak{F}_{2}$.

Отсюда $A_{1}=B_{1_{1}} \times \cdots \times B_{t_{1}} \in \mathfrak{F}_{1}, A_{2}=B_{1_{2}} \times \cdots \times B_{t_{2}} \in \mathfrak{F}_{2}$. Покажем, что

$$
A \in \mathfrak{F}=\left(l^{\infty} \text { fit } A_{1}\right) \vee^{\infty}\left(l^{\infty} \text { fit } A_{2}\right)
$$

Пусть $h$ - минимальная $l^{\infty}$-значная $H$-функция класса $\mathfrak{F}$ и $f-l^{\infty}$-значная $H$-функция класса $\mathfrak{F}$ такая, что $f(p)=h(p) \mathfrak{N}_{p}$ для любого $p \in \mathbb{P}$. Пусть $i \in\{1, \ldots, t\}$. Покажем, что $F^{p_{i}}(A) \in f\left(p_{i}\right)$. Сначала установим, что $A_{i_{1}}, A_{i_{2}} \in f\left(p_{i}\right)$. Допустим, что $A_{i_{1}} \notin f\left(p_{i}\right)$. Тогда поскольку $f\left(p_{i}\right)$ - класс Локетта [13], согласно [1, гл. Х, свойство $2.1 \mathrm{a})]\left(B_{i_{1}}\right)_{f\left(p_{i}\right)}=K_{1}$, где $K_{1}$ - база регулярного сплетения $\left(A_{i_{1}}\right)_{f\left(p_{i}\right)}$ 々 $Z_{p_{i}}$. Ввиду свойств сплетений (см., например, [1, гл. А, 18.2 d)])

$$
B_{i_{1}} /\left(B_{i_{1}}\right)_{f\left(p_{i}\right)}=B_{i_{1}} / K_{1} \simeq\left(A_{i_{1}} /\left(A_{i_{1}}\right)_{f\left(p_{i}\right)}\right) \curlywedge Z_{p_{i}}
$$

Значит, $p_{i}$ делит порядок $B_{i_{1}} /\left(B_{i_{1}}\right)_{f\left(p_{i}\right)}$.

$\mathrm{C}$ другой стороны, так как $B_{i_{1}} \in \mathfrak{F}$, то

$$
B_{i_{1}} \in \mathfrak{G}_{\pi(\mathfrak{F})} \cap\left(\bigcap_{p \in \pi(\mathfrak{F})} f(p) \mathfrak{G}_{p^{\prime}}\right)
$$


и, в частности, $B_{i_{1}} \in f\left(p_{i}\right) \mathfrak{G}_{p_{i}^{\prime}}$. Следовательно, $B_{i_{1}} /\left(B_{i_{1}}\right)_{f\left(p_{i}\right)} \in \mathfrak{G}_{p_{i}^{\prime}}$. Противоречие. Значит, $A_{i_{1}} \in f\left(p_{i}\right)$. Аналогично, $A_{i_{2}} \in f\left(p_{i}\right)$. Поэтому $l^{\infty}$ fit $\left(A_{i_{1}}, A_{i_{2}}\right) \subseteq f\left(p_{i}\right)$. Очевидно, $l^{\infty}$ fit $\left(A_{i_{1}}, A_{i_{2}}\right)=\left(l^{\infty}\right.$ fit $\left.A_{i_{1}}\right) \vee^{\infty}\left(l^{\infty}\right.$ fit $\left.A_{i_{2}}\right)$. Следовательно, $F^{p_{i}}(A) \in f\left(p_{i}\right)$.

Если же $A \in \mathfrak{F}_{1} \wedge^{\infty} \mathfrak{F}_{2}=\mathfrak{F}_{1} \cap \mathfrak{F}_{2}$, то $A \in\left(l^{\infty}\right.$ fit $\left.A\right) \wedge^{\infty}\left(l^{\infty}\right.$ fit $\left.A\right)$. Таким образом, мы завершили доказательство теоремы при $r=1$.

Пусть терм $\omega$ имеет $r>1$ вхождений символов из $\left\{\cap, \vee^{\infty}\right\}$, и для термов с меньшим числом вхождений лемма верна. Пусть $\omega$ имеет вид

$$
\omega_{1}\left(x_{i_{1}}, \ldots, x_{i_{a}}\right) \Delta \omega_{2}\left(x_{j_{1}}, \ldots, x_{j_{b}}\right),
$$

где $\Delta \in\left\{\cap, \vee^{\infty}\right\}$ и $\left\{x_{i_{1}}, \ldots, x_{i_{a}}\right\} \cup\left\{x_{j_{1}}, \ldots, x_{j_{b}}\right\}=\left\{x_{1}, \ldots, x_{m}\right\}$.

Через $\mathfrak{H}_{1}$ обозначим класс Фиттинга $\omega_{1}\left(\mathfrak{F}_{i_{1}}, \ldots, \mathfrak{F}_{i_{a}}\right)$, через $\mathfrak{H}_{2}-$ класс Фиттинга $\omega_{2}\left(\mathfrak{F}_{j_{1}}, \ldots, \mathfrak{F}_{j_{b}}\right)$. Тогда по доказанному вьше найдутся групшы $A_{1} \in \mathfrak{H}_{1}, A_{2} \in \mathfrak{H}_{2}$ такие, что $A \in l^{\infty}$ fit $A_{1} \Delta l^{\infty}$ fit $A_{2}$. Поскольку число операций в терме $\omega_{1}$ меньше $r$, по индукции найдутся группы $B_{1} \in \mathfrak{F}_{i_{1}}, \ldots, B_{a} \in \mathfrak{F}_{i_{a}}$ такие, что

$$
A_{1} \in \omega_{1}\left(l^{\infty} \text { fit } B_{1}, \ldots, l^{\infty} \text { fit } B_{a}\right) .
$$

Аналогично, найдутся групшы $C_{1} \in \mathfrak{F}_{j_{1}}, \ldots, C_{b} \in \mathfrak{F}_{j_{b}}$ такие, что

$$
A_{2} \in \omega_{2}\left(l^{\infty} \text { fit } C_{1}, \ldots, l^{\infty} \text { fit } C_{b}\right) .
$$

Пусть $x_{i_{t+1}}, \ldots, x_{i_{a}} \in\left\{x_{j_{1}}, \ldots, x_{j_{b}}\right\}$ и, вместе с тем, $\left\{x_{i_{1}}, \ldots, x_{i_{t}}\right\} \cap\left\{x_{j_{1}}, \ldots, x_{j_{b}}\right\}=\varnothing$. Пусть

$$
D_{i_{k}}= \begin{cases}B_{k}, & \text { если } k<t+1, \\ B_{k} \times C_{q}, & \text { где } x_{i_{k}}=x_{j_{q}} \text { для некоторого } q \in\{1, \ldots, b\} \text { при } k \geqslant t+1 .\end{cases}
$$

Пусть $D_{j_{k}}=C_{k}$, если $x_{j_{k}} \notin\left\{x_{i_{t+1}}, \ldots, x_{i_{a}}\right\}$. Через $\mathfrak{M}_{p}$ обозначим класс $l^{\infty}$ fit $D_{i_{p}}$, где $p=1, \ldots, a$; через $\mathfrak{X}_{c}-$ класс $l^{\infty}$ fit $D_{j_{c}}$, где $c=1, \ldots, b$.

Итак,

$A_{1} \in \omega_{1}\left(l^{\infty}\right.$ fit $B_{1}, \ldots, l^{\infty}$ fit $\left.B_{a}\right) \subseteq \omega_{1}\left(l^{\infty}\right.$ fit $D_{i_{1}}, \ldots, l^{\infty}$ fit $\left.D_{i_{a}}\right)=\omega_{1}\left(\mathfrak{M}_{1}, \ldots, \mathfrak{M}_{a}\right)$, $A_{2} \in \omega_{2}\left(l^{\infty}\right.$ fit $C_{1}, \ldots, l^{\infty}$ fit $\left.C_{b}\right) \subseteq \omega_{2}\left(l^{\infty}\right.$ fit $D_{j_{1}}, \ldots, l^{\infty}$ fit $\left.D_{j_{b}}\right)=\omega_{2}\left(\mathfrak{X}_{1}, \ldots, \mathfrak{X}_{b}\right)$.

Значит, найдутся классы Фиттинга $\mathfrak{R}_{1}, \ldots, \mathfrak{R}_{m}$ такие, что

$$
A \in \omega_{1}\left(\mathfrak{R}_{i_{1}}, \ldots, \mathfrak{R}_{i_{a}}\right) \Delta \omega_{2}\left(\mathfrak{R}_{j_{1}}, \ldots, \mathfrak{R}_{j_{b}}\right)=\omega\left(\mathfrak{R}_{1}, \ldots, \mathfrak{R}_{m}\right),
$$

где $\mathfrak{R}_{i}=l^{\infty}$ fit $K_{i}, K_{i} \in \mathfrak{F}_{i}$. Лемма доказана.

Лемма 7. Пусть $\omega\left(x_{1}, \ldots, x_{m}\right)-$ терм сигнатуры $\left\{\cap, \vee^{\infty}\right\}, f_{i}-$ внутренняя

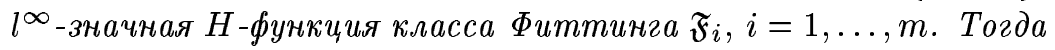

$$
\omega\left(\mathfrak{F}_{1}, \ldots, \mathfrak{F}_{m}\right)=\operatorname{LR}\left(\omega\left(f_{1}, \ldots, f_{m}\right)\right) .
$$


ДоКАЗАТЕЛЬСТВО. Проведем индукцию по числу $r$ вхождений символов из $\left\{\cap, \vee^{\infty}\right\}$ в терм $\omega$. Пусть

$$
\omega\left(x_{1}, \ldots, x_{m}\right)=\omega_{1}\left(x_{i_{1}}, \ldots, x_{i_{a}}\right) \Delta \omega_{2}\left(x_{j_{1}}, \ldots, x_{j_{b}}\right)
$$

где $\Delta \in\left\{\cap, \vee^{\infty}\right\}$ и $\left\{x_{i_{1}}, \ldots, x_{i_{a}}\right\} \cup\left\{x_{j_{1}}, \ldots, x_{j_{b}}\right\}=\left\{x_{1}, \ldots, x_{m}\right\}$. Допустим, что для термов $\omega_{1}$ и $\omega_{2}$ лемма верна. Тогда

$$
\omega_{1}\left(\mathfrak{F}_{i_{1}}, \ldots, \mathfrak{F}_{i_{a}}\right)=\operatorname{LR}\left(\omega_{1}\left(f_{i_{1}}, \ldots, f_{i_{a}}\right)\right), \quad \omega_{2}\left(\mathfrak{F}_{j_{1}}, \ldots, \mathfrak{F}_{j_{b}}\right)=\operatorname{LR}\left(\omega_{2}\left(f_{j_{1}}, \ldots, f_{j_{b}}\right)\right)
$$

Понятно, что $\omega_{1}\left(f_{i_{1}}, \ldots, f_{i_{a}}\right)$ и $\omega_{2}\left(f_{j_{1}}, \ldots, f_{j_{b}}\right)$ - внутренние $l^{\infty}$-значные $H$-функции классов Фиттинга $\omega_{1}\left(\mathfrak{F}_{i_{1}}, \ldots, \mathfrak{F}_{i_{a}}\right)$ и $\omega_{2}\left(\mathfrak{F}_{j_{1}}, \ldots, \mathfrak{F}_{j_{b}}\right)$ соответственно. Значит, по индукции

$$
\begin{aligned}
\omega\left(\mathfrak{F}_{1}, \ldots, \mathfrak{F}_{m}\right) & =\omega_{1}\left(\mathfrak{F}_{i_{1}}, \ldots, \mathfrak{F}_{i_{a}}\right) \Delta \omega_{2}\left(\mathfrak{F}_{j_{1}}, \ldots, \mathfrak{F}_{j_{b}}\right) \\
& =\operatorname{LR}\left(\omega_{1}\left(f_{i_{1}}, \ldots, f_{i_{a}}\right) \Delta \omega_{2}\left(f_{j_{1}}, \ldots, f_{j_{b}}\right)\right)=\operatorname{LR}\left(\omega\left(f_{1}, \ldots, f_{m}\right)\right),
\end{aligned}
$$

где $\Delta \in\left\{\cap, \vee^{\infty}\right\}$. Лемма доказана.

Элемент $c$ полной решетки $L$ назьвается компактным, если для любого подмножества $X \subseteq L$ из неравенства $c \leqslant \sup _{L} X$ вытекает существование конечного подмножества $X_{0} \subseteq X$ такого, что $c \leqslant \sup _{L} X_{0}$.

Лемма 8. Пусть $\mathfrak{F}=l^{\infty}$ fit $G$, где группа $G$ разрешима. Тогда $\mathfrak{F}$ является компактным әлементом в решетке $l^{\infty}$.

ДокАЗАТЕЛЬСТво. Индукцией по нильпотентной длине групшы $G$ покажем, что $\mathfrak{F}$ является компактным элементом в $l^{\infty}$. Пусть $\mathfrak{F} \subseteq \mathfrak{M}=\vee^{\infty}\left(\mathfrak{F}_{i} \mid i \in I\right)$, где $\mathfrak{F}_{i}$-тотально локальный класс Фиттинга.

Если $l(G)=1$, то $G=P_{1} \times \cdots \times P_{k}$, где $P_{i}-$ силовская $p_{i}$-подгруппа групшы $G$. Значит, найдутся индексы $j_{1}, \ldots, j_{k} \in I$ такие, что $p_{i} \in \pi\left(\mathfrak{F}_{j_{i}}\right)$, т.е. $P_{i} \in \mathfrak{F}_{j_{i}}$. Поэтому $G \in \mathfrak{F}_{j_{1}} \vee^{\infty} \cdots \vee^{\infty} \mathfrak{F}_{j_{k}}$. Следовательно, $\mathfrak{F} \subseteq \mathfrak{F}_{j_{1}} \vee^{\infty} \cdots \vee^{\infty} \mathfrak{F}_{j_{k}}$.

Пусть $l(G)>1$ и все тотально локальные классы Фиттинга вида $l^{\infty}$ fit $A$, где $A-$ разрешимая группа и $l(A)<l(G)$, являются компактньми элементами в решетке $l^{\infty}$. Пусть $f_{i}$ - минимальная $l^{\infty}$-значная $H$-функция класса $\mathfrak{F}_{i}, f$-минимальная $l^{\infty}$-значная $H$-функция класса $\mathfrak{F}$ и $m$ - минимальная $l^{\infty}$-значная $H$-функция класса $\mathfrak{M}$. Тогда по лемме $1 f(p)=l^{\infty} \operatorname{fit}\left(F^{p}(G)\right)$ при всех $p \in \pi(G)$ и $f(p)=\varnothing$ при всех $p \in \mathbb{P} \backslash \pi(G)$. Кроме того, из леммы 1 вытекает, что $f \leqslant m$. Согласно лемме $2 m=\vee^{\infty}\left(f_{i} \mid i \in I\right)$. Поскольку $l\left(F^{p}(G)\right)<l(G)$, по индукции для каждого $p \in \pi(G)$ найдутся индексы $i_{1}, \ldots, i_{t} \in I$ такие, что

$$
F^{p}(G) \in f_{i_{1}}(p) \vee^{\infty} \cdots \vee^{\infty} f_{i_{t}}(p)
$$

Поскольку $|\pi(G)|<\infty$, из последнего вытекает, что найдутся индексы $j_{1}, \ldots, j_{k} \in I$ такие, что $G \in \mathfrak{F}_{j_{1}} \vee^{\infty} \ldots \vee^{\infty} \mathfrak{F}_{j_{k}}$. Таким образом, $\mathfrak{F} \subseteq \mathfrak{F}_{j_{1}} \vee^{\infty} \ldots \vee^{\infty} \mathfrak{F}_{j_{k}}$. Значит, $\mathfrak{F}-$ компактньй элемент в решетке $l^{\infty}$. Лемма доказана.

Символом $L^{\infty}(\mathfrak{F})$ обозначается (см. [6]) решетка всех тотально локальных подклассов Фиттинга из $\mathfrak{F}$. 
Лемма 9. Пусть $\mathfrak{F}-$ тотально локальный класс Фиттинга. Тогда при любом натуральном $k \geqslant 2$ решетки $L^{\infty}\left(\mathfrak{F N}^{k-1}\right)$ и $L^{\infty}\left(\mathfrak{F N}^{k}\right)$ порождают одно и то же многообразие решеток.

ДокАЗАТЕЛЬСтво. Зафиксируем некоторое тождество

$$
\omega_{1}\left(x_{i_{1}}, \ldots, x_{i_{a}}\right)=\omega\left(x_{j_{1}}, \ldots, x_{j_{b}}\right)
$$

сигнатуры $\left\{\cap, \vee^{\infty}\right\}$.

Если тождество $(*)$ выполняется в решетке $L^{\infty}\left(\mathfrak{F N}^{k}\right)$, то оно выполняется и в любой подрешетке решетки $L^{\infty}\left(\mathfrak{F N}^{k}\right)$. Поэтому тождество $(*)$ справедливо в решетке $L^{\infty}\left(\mathfrak{F N}^{k-1}\right)$.

Пусть теперь тождество $(*)$ вьполняется в решетке $L^{\infty}\left(\mathfrak{F} \mathfrak{N}^{k-1}\right)$ и пусть $\mathfrak{F}_{i_{1}}, \ldots, \mathfrak{F}_{i_{a}}$, $\mathfrak{F}_{j_{1}}, \ldots, \mathfrak{F}_{j_{b}}$ - произвольные классы Фиттинга из $L^{\infty}\left(\mathfrak{F N}^{k}\right)$. Пусть $f_{i_{c}}$ - минимальная $l^{\infty}$-значная $H$-функция класса $\mathfrak{F}_{i_{c}}, c=1, \ldots, a$, и пусть $f_{j_{d}}$ - минимальная $l^{\infty}$-значная $H$-функция класса $\mathfrak{F}_{j_{d}}, d=1, \ldots, b$. По лемме 7

$$
\omega_{1}\left(\mathfrak{F}_{i_{1}}, \ldots, \mathfrak{F}_{i_{a}}\right)=\operatorname{LR}\left(\omega_{1}\left(f_{i_{1}}, \ldots, f_{i_{a}}\right)\right), \quad \omega_{2}\left(\mathfrak{F}_{j_{1}}, \ldots, \mathfrak{F}_{j_{b}}\right)=\operatorname{LR}\left(\omega_{2}\left(f_{j_{1}}, \ldots, f_{j_{b}}\right)\right) .
$$

Заметим, что для любого $p \in \mathbb{P}$ классы

$$
f_{i_{1}}(p), \ldots, f_{i_{a}}(p), f_{j_{1}}(p), \ldots, f_{j_{b}}(p)
$$

принадлежат решетке $L^{\infty}\left(\mathfrak{F N}^{k-1}\right)$. Следовательно,

$\omega_{1}\left(f_{i_{1}}, \ldots, f_{i_{a}}\right)(p)=\omega_{1}\left(f_{i_{1}}(p), \ldots, f_{i_{a}}(p)\right)=\omega_{2}\left(f_{j_{1}}(p), \ldots, f_{j_{b}}(p)\right)=\omega_{2}\left(f_{j_{1}}, \ldots, f_{j_{b}}\right)(p)$.

Поэтому $\omega_{1}\left(\mathfrak{F}_{i_{1}}, \ldots, \mathfrak{F}_{i_{a}}\right)=\omega_{2}\left(\mathfrak{F}_{j_{1}}, \ldots, \mathfrak{F}_{j_{b}}\right)$. Таким образом, тождество $(*)$ справедливо в решетке $L^{\infty}\left(\mathfrak{F N}^{k}\right)$. Лемма доказана.

Лемма 10. Пусть $\eta$ - такая подрешетка решетки разрешимых тотально локальных классов Фиттинга, которая со всяким своим классом Фиттинга $\mathfrak{F}$ содержит и все его однопорожденные тотально локальные подклассы Фиттинга. Тогда тождество $\omega_{1}=\omega_{2}$ сигнатуры $\left\{\cap, \vee^{\infty}\right\}$ истинно в $\eta$, если оно выполняется для всех однопорожденных тотально локальных классов Фиттинга из $\eta$.

ДокАЗАТЕЛьство. Пусть $x_{i_{1}}, \ldots, x_{i_{a}}$-переменные, входящиев терм $\omega_{1} ; x_{j_{1}}, \ldots, x_{j_{b}}$ - переменные, входяшие в терм $\omega_{2}$, и пусть $\mathfrak{F}_{i_{1}}, \ldots, \mathfrak{F}_{i_{a}}, \mathfrak{F}_{j_{1}}, \ldots, \mathfrak{F}_{j_{b}} \in \eta$. Покажем, что

$$
\mathfrak{F}=\omega_{1}\left(\mathfrak{F}_{i_{1}}, \ldots, \mathfrak{F}_{i_{a}}\right) \subseteq \omega_{2}\left(\mathfrak{F}_{j_{1}}, \ldots, \mathfrak{F}_{j_{b}}\right)=\mathfrak{M} .
$$

Пусть $x_{j_{1}}, \ldots, x_{j_{t}} \in\left\{x_{i_{1}}, \ldots, x_{i_{a}}\right\}$; но $\left\{x_{j_{t+1}}, \ldots, x_{j_{b}}\right\} \cap\left\{x_{i_{1}}, \ldots, x_{i_{a}}\right\}=\varnothing$. Пусть $A \in \mathfrak{F}$. Тогда по лемме 6 найдутся грушы $A_{i_{1}}, \ldots, A_{i_{a}}$ такие, что $A_{i_{k}} \in \mathfrak{F}_{i_{k}}$ для всех $k \in\{1, \ldots, a\}$ и

$$
A \in \mathfrak{S} \cap \omega_{1}\left(l^{\infty} \text { fit } A_{i_{1}}, \ldots, l^{\infty} \text { fit } A_{i_{a}}\right) .
$$

Пусть $\mathfrak{H}_{i_{k}}=l^{\infty}$ fit $A_{i_{k}}$ и

$\mathfrak{H}_{j_{k}}= \begin{cases}\mathfrak{H}_{i_{c}}, & \text { где } x_{j_{k}}=x_{i_{c}} \text { для некоторого } c \in\{1, \ldots, a\} \text { при всех } k \in\{1, \ldots, t\}, \\ l^{\infty} \text { fit } B_{j_{k}} & \text { для некоторой групшы } B_{j_{k}} \in \mathfrak{F}_{j_{k}} \text { при } k>t .\end{cases}$

По условию $\omega_{1}\left(\mathfrak{H}_{i_{1}}, \ldots, \mathfrak{H}_{i_{a}}\right)=\omega_{2}\left(\mathfrak{H}_{j_{1}}, \ldots, \mathfrak{H}_{j_{b}}\right) \subseteq \mathfrak{M}$. Значит, $A \in \mathfrak{M}$. Итак, $\mathfrak{F} \subseteq \mathfrak{M}$. Аналогично доказьвается обратное включение. Лемма доказана.

Напомним [6], что если классы групп $\mathfrak{M}$ и $\mathfrak{H}$ таковы, что $\mathfrak{M} \cap \mathfrak{H}=(1)$, то через $\mathfrak{M} \oplus \mathfrak{H}$ обозначается совокупность всех групп вида $\{A \times B \mid A \in \mathfrak{M}, B \in \mathfrak{H}\}$. 
Теорема. Решетка всех разрешимых тотально локальных классов Фиттинга алгебраична, дистрибутивна и каждый ее әлемент, отличный от (1) $u \mathfrak{S}$, не дополняем в ней.

ДокАЗАТЕЛЬСтво. Покажем, что решетка $L^{\infty}(\mathfrak{S})$ алгебраична. Очевидно, любой тотально локальный класс Фиттинга есть объединение своих однопорожденных тотально локальных подклассов $\Phi$ иттинга в решетке $l^{\infty}$. Пусть $\mathfrak{F}=l^{\infty}$ fit $G$, где группа $G$ разрешима. По лемме $8 \mathfrak{F}$-компактньй элемент в решетке $l^{\infty}$. Значит, $\mathfrak{F}$ будет компактньм элементом и в подрешетке $L^{\infty}(\mathfrak{S})$ решетки $l^{\infty}$. Следовательно, решетка всех разрешимых тотально локальных классов Фиттинга алгебраична, и ее компактньми элементами являются однопорожденные тотально локальные классы Фиттинга.

Докажем теперь, что решетка $L^{\infty}(\mathfrak{S})$ дистрибутивна. Но прежде индукцией по $r$ покажем дистрибутивность решетки $L^{\infty}\left(\mathfrak{N}^{r}\right)$. Решетка $L^{\infty}(\mathfrak{N})$, очевидно, дистрибутивна. Пусть $r>1$ и решетка $L^{\infty}\left(\mathfrak{N}^{r-1}\right)$ дистрибутивна. Тогда по лемме 9 решетка $L^{\infty}\left(\mathfrak{N}^{r}\right)$ также дистрибутивна.

Пусть теперь $\mathfrak{F}=l^{\infty}$ fit $G$, где $l(G)=r$. Тогда $G \in \mathfrak{N}^{r}$ и поэтому $L^{\infty}\left(l^{\infty}\right.$ fit $\left.G\right) \subseteq$ $L^{\infty}\left(\mathfrak{N}^{r}\right)$. Следовательно, решетка $L^{\infty}\left(l^{\infty}\right.$ fit $\left.G\right)$ дистрибутивна. Значит, ввиду леммы 10 решетка всех разрешимых тотально локальных классов Фиттинга дистрибутивна.

Докажем теперь, что каждьй разрешимьй тотально локальный класс Фиттинга, отличный от (1) и $\mathfrak{S}$, не дополняем в решетке $L^{\infty}(\mathfrak{S})$. Пусть $\mathfrak{M} \neq(1)$ и $\mathfrak{M} \neq \mathfrak{S}$ - разрешимый тотально локальньй класс Фиттинга и пусть $\mathfrak{H}-$ дополнение к $\mathfrak{M}$ в решетке $L^{\infty}(\mathfrak{S})$. Тогда $\mathfrak{S}=\mathfrak{M} \vee^{\infty} \mathfrak{H}$ и $\mathfrak{M} \cap \mathfrak{H}=(1)$.

Покажем, что $\mathfrak{M} \vee \infty \mathfrak{H}=\mathfrak{M} \vee \mathfrak{H}$. Рассмотрим класс Фиттинга $\mathfrak{F}=\mathfrak{M} \vee \mathfrak{H}$. Так как $\mathfrak{M} \cap \mathfrak{H}=(1)$, то $\mathscr{K}(\mathfrak{M}) \cap \mathscr{K}(\mathfrak{H})=\varnothing$, где $\mathscr{K}(\mathfrak{M})$ - набор всех композиционных факторов групп из $\mathfrak{M}, \mathscr{K}(\mathfrak{H})$ - набор всех композиционных факторов групп из $\mathfrak{H}$. Следовательно, по лемме 4 [14] $\mathfrak{F}=\mathfrak{M} \oplus \mathfrak{H}$. Покажем, что класс $\mathfrak{M} \oplus \mathfrak{H}$ тотально локален. Пусть $m$ и $h$ - минимальные $l^{\infty}$-значные $H$-функции классов $\mathfrak{M}$ и $\mathfrak{H}$ соответственно. Пусть $f-$ $H$-функция такая, что

$$
f(p)= \begin{cases}m(p), & \text { если } p \in \pi(\mathfrak{M}), \\ h(p), & \text { если } p \in \pi(\mathfrak{H}), \\ \varnothing, & \text { если } p \in \mathbb{P} \backslash(\pi(\mathfrak{M}) \cup \pi(\mathfrak{H}))\end{cases}
$$

Покажем, что $\mathfrak{F}=\operatorname{LR}(f)$. Пусть $G$ - группа минимального порядка из $\operatorname{LR}(f) \backslash \mathfrak{F}$. Тогда $G$ - комонолитическая группа и ее комонолит $M=G_{\mathfrak{F}}$. Поскольку $G \in \operatorname{LR}(f)$, то $F^{p}(G) \in f(p)$ для всех $p \in \pi(G)$. Следовательно, если $p \in \pi(G)$, то по построению $H$-функции $f$ либо $f(p)=m(p) \neq \varnothing$, либо $f(p)=h(p) \neq \varnothing$. Значит, $\pi(G) \subseteq \pi(\mathfrak{M}) \cup \pi(\mathfrak{H})$.

Пусть $p \in \pi(G / M)$. Тогда $p \in \pi(\mathfrak{M}) \cup \pi(\mathfrak{H})$. Пусть теперь $p \in \pi(\mathfrak{M})$. Следовательно, $G / M-p$-группа и $F^{p}(G)=O^{p}(G) \in f(p)=m(p)$. Значит, по лемме $4 G \in \mathfrak{M} \subseteq \mathfrak{F}$. Противоречие. Таким образом, $\operatorname{LR}(f) \subseteq \mathfrak{F}$.

Допустим, что обратное включение неверно и $G$ - групша минимального порядка из $\mathfrak{F} \backslash \operatorname{LR}(f)$. Тогда $G$ - комонолитическая группа. Поэтому либо $G \in \mathfrak{M}$, либо $G \in \mathfrak{H}$. Пусть $G \in \mathfrak{M}=\operatorname{LR}(m)$. Значит, $F^{p}(G) \in m(p)=f(p)$ для всех $p \in \pi(G)$. Следовательно, $G \in \operatorname{LR}(f)$. Значит, $\mathfrak{F} \subseteq \operatorname{LR}(f)$. Таким образом, $\mathfrak{F}=\operatorname{LR}(f)$ - тотально локальньй 
класс Фиттинга. Но $\mathfrak{M} \vee \mathfrak{H} \subseteq \mathfrak{F}$. Значит, $\mathfrak{M} \vee^{\infty} \mathfrak{H}=\mathfrak{M} \oplus \mathfrak{H}=\mathfrak{M} \vee \mathfrak{H}$. Следовательно, для любой комонолитической групшы $G \in \mathfrak{S}$ имеет место одно из двух: $G \in \mathfrak{M}$ или $G \in \mathfrak{H}$.

Пусть $Z_{p}$ и $Z_{q}$ - некоторые групшы порядков $p$ и $q$ соответственно, где $p \in \pi(\mathfrak{M})$, $q \in \pi(\mathfrak{H})$. Пусть $r \in \mathbb{P} \backslash\{p, q\}$. Тогда ввиду [1, гл. В, следствие 10.7$]$ группа $A=Z_{p} \times Z_{q}$ обладает простым точным модулем $P$ над полем из $r$ элементов $\mathbb{F}_{r}$. Пусть $B=[P] A$. Тогда $B \in \mathfrak{S}$ и, вместе с тем, $B \notin \mathfrak{M}$, поскольку $q \notin \pi(\mathfrak{M})$, и $B \notin \mathfrak{H}$, поскольку $p \notin \pi(\mathfrak{H})$. Но группа $B$ комонолитична и, значит, либо $B \in \mathfrak{M}$, либо $B \in \mathfrak{H}$. Противоречие. Итак, каждьй ненулевой и неединичньй элемент решетки всех разрешимых тотально локальных классов Фиттинга не дополняем в ней. Теорема доказана.

Пусть $L$-произвольная решетка. Псевдодополнением әлемента а относительно элемента $b$ назьвается [15] наибольший из элементов $x$ решетки $L$, удовлетворяющих неравенству $a \wedge x \leqslant b$. Псевдодополнение элемента $a$ относительно элемента $b$ обозначается через $a * b$. Псевдодополнением әлемента а в решетке с нулем назьвается относительное псевдодополнение $a * 0$. Решетка $c$ псевдодополнениями - это такая решетка с нулем, каждый элемент которой имеет псевдодополнение.

Согласно следствию 2 теоремы 1 из книги [16, гл. II, с. 151] из доказанной теоремы вытекает

СледствиЕ 1. Решетка всех разрешимых тотально локальных классов Фиттинга является решеткой с псевдодополнениями.

Напомним, что представление элемента $a$ в виде $x_{0} \vee \cdots \vee x_{n-1}$ назьвается сократимым.м[16], если $a=x_{0} \vee \cdots \vee x_{i-1} \vee x_{i+1} \vee \cdots \vee x_{n-1}$ для некоторого $0 \leqslant i<n$; в противном случае оно назьвается несократимым.

Тотально локальный класс Фиттинга $\mathfrak{F}$ назьвается $l^{\infty}$-неприводимым.м [2], если класс $\mathfrak{F}$ нельзя представить в виде $\mathfrak{F}=\vee^{\infty}\left(\mathfrak{F}_{i} \mid i \in I\right)$, где $\left\{\mathfrak{F}_{i} \mid i \in I\right\}$ - набор всех собственных тотально локальных подклассов Фиттинга из $\mathfrak{F}$.

СлЕДСТВИЕ 2. Пусть $\mathfrak{F}$ - разрешимый однопорожденный тотально локальный класс Фиттинга. Тогда $\mathfrak{F}$ обладает единственным представлением в виде несократимого обвединения $\mathfrak{F}_{1} \vee^{\infty} \ldots \vee^{\infty} \mathfrak{F}_{t}$ некоторых своих тотально локальных $l^{\infty}$-неприводимых подклассов Фиттинга $\mathfrak{F}_{1}, \ldots, \mathfrak{F}_{t}$.

ДокАЗАТЕЛЬСТво. Ввиду следствия 13 теоремы 9 из книги [16, гл. II] для доказательства данного следствия достаточно лишь установить, что решетка $L^{\infty}(\mathfrak{F})$ всех тотально локальных подклассов Фиттинга произвольного разрешимого однопорожденного тотально локального класса Фиттинга $\mathfrak{F}=l^{\infty}$ fit $G$ конечна. Проведем индукцию по нильпотентной длине группы $G$.

Если $l(G)=1$, то каждый неединичньй тотально локальный подкласс Фиттинга из $\mathfrak{F}$ имеет вид $\mathfrak{N}_{\pi}$, где $\pi \subseteq \pi(\mathfrak{F})$. Значит, в $\mathfrak{F}$ имеется лишь конечное множество тотально локальных подклассов Фиттинга.

Пусть $l(G)>1$ и для всех тотально локальных классов Фиттинга вида $l^{\infty}$ fit $A$, где $l(A)<l(G)$, решетка $L^{\infty}\left(l^{\infty}\right.$ fit $\left.A\right)$ конечна. Пусть $\mathfrak{M}$ - произвольный тотально локальньй подкласс Фиттинга из $\mathfrak{F}$ и пусть $m$ и $f$-минимальные $l^{\infty}$-значные $H$-функции классов $\mathfrak{M}$ и $\mathfrak{F}$ соответственно. Тогда из леммы 1 вытекает, что $m \leqslant f$. Кроме того, ввиду этой же леммы при любом $p \in \pi(\mathfrak{F})$ имеет место равенство

$$
f(p)=l^{\infty} \operatorname{fit}\left(F^{p}(A) \mid A \in \mathfrak{F}\right) .
$$


Поскольку $l\left(F^{p}(G)\right)<l(G)$, по индукции решетка $L^{\infty}(f(p))$ конечна. Так как при этом конечно и множество $\pi(\mathfrak{F})$, в $\mathfrak{F}$ имеется лишь конечное множество тотально локальных подклассов Фиттинга. Следствие доказано.

Для любых двух тотально локальных классов Фиттинга $\mathfrak{M}$ и $\mathfrak{H}$, где $\mathfrak{M} \subseteq \mathfrak{H}$, через $\mathfrak{H} /{ }^{\infty} \mathfrak{M}$ обозначается [6] решетка тотально локальных классов Фиттинга, заключенных между $\mathfrak{M}$ и $\mathfrak{H}$.

Так как всякая дистрибутивная решетка является модулярной, из доказанной теоремы вытекает

СлЕДСТВИЕ 3. Для любых двух разрешимых тотально локальных классов Фиттинга $\mathfrak{M}$ и $\mathfrak{H}$ справедлив решеточный изоморфизм

$$
\mathfrak{M} \vee^{\infty} \mathfrak{H} /{ }^{\infty} \mathfrak{M} \simeq \mathfrak{H} /{ }^{\infty} \mathfrak{H} \cap \mathfrak{M}
$$

СледствИЕ 4 [6, теорема 4.1.7]. Решетка всех разрешимых тотально локальных формачий дистрибутивна.

ДокАЗАТЕльство. Покажем, что каждьй разрешимый тотально локальньй класс Фиттинга $\mathfrak{F}$ наследственен. Не теряя общности, мы можем предполагать, что $\mathfrak{F}=l^{\infty}$ fit $G$ для некоторой разрешимой группы $G$. Если $G$ - нильпотентная группа, то утверждение очевидно. Пусть нильпотентная длина $t$ группы $G$ больше 1 . Тогда если $f$ - минимальная $l^{\infty}$-значная $H$-функция класса $\mathfrak{F}$, то поскольку $l\left(F^{p}(G)\right)<t$, ввиду соображений индукции для любого $p \in \pi(G)$ класс Фиттинга $f(p)=l^{\infty} \operatorname{fit}\left(F^{p}(G)\right)$ наследственен. Значит, класс $\mathfrak{F}$ наследственен, и поэтому ввиду результатов работы [17] класс $\mathfrak{F}$ является тотально локальной формацией. Следовательно, каждьй разрешимьй тотально локальный класс Фиттинга является тотально локальной формацией.

Рассуждая аналогичным образом, легко убедиться, что каждая разрешимая тотально локальная формация является наследственным классом Фиттинга. Но по теореме работы [7] каждый разрешимый наследственный класс Фиттинга является тотально локальным классом Фиттинга. Значит, каждая разрешимая тотально локальная формация является тотально локальным классом Фиттинга.

Таким образом, решетки $L^{\infty}(\mathfrak{S})$ и $L_{\infty}(\mathfrak{S})$ совпадают. Поэтому ввиду доказанной теоремы решетка всех разрешимых тотально локальных формаций дистрибутивна. Следствие доказано.

Предложенная в настоящей работе техника доказательств и методы рассуждений тесно привязаны к условию разрешимости. Что же касается неразрешимого случая, то мы не можем ничего сказать даже о модулярности решетки всех тотально локальных классов Фиттинга.

Вопрос. Дистрибутивна (или хотя бы модулярна) ли решетка всех тотально локальных классов Фиттинга?

Аналогичный вопрос для тотально локальных формаций приведен в монографии [6, вопрос 4.2.14]. 


\section{СПИСОК ЦИТИРОВАННОЙ ЛИТЕРАТУРЫ}

[1] Doerk K., Hawkes T. Finite Soluble Groups. Berlin-New York: Walter de Gruyter, 1992.

[2] Скиба А. Н., Шеметков Л. А. Кратно $\omega$-локальные формации и классы Фиттинга конечных групп. Препринты Гомельского госуниверситета, 1997.

[3] Скиба А.Н. О локальных формациях длины 5 // Арифметическое и подгрупповое строение конечных групп. Минск: Наука и техника, 1986. С. 135-149.

[4] Hawkes T. O. Sceletal classes of soluble groups // Arch. Math. 1971. V. 22. № 6. P. 577-589.

[5] Шеметков Л. А., Скиба А. Н. Формации алгебраических систем. М.: Наука, 1989.

[6] Скиба А.Н. Алгебра формаций. Минск: Беларуская навука, 1997.

[7] Воробьев Н. Т. О предположении Хоукса для радикальных классов // Сиб. матем. ж. 1996. Т. 37. № 6. С. 1296-1302.

[8] Каморников С.Ф. О двух задачах из "Коуровской тетради" // Матем. заметки. 1994. T. 55. №6. C. 59-63.

[9] Семенчук В. Н. Описание разрешимых минимальных не $\mathfrak{F}$-групп для произвольной тотально локальной формации $\mathfrak{F} / /$ Матем. заметки. 1988. Т. 43. № 4. С. 452-459.

[10] Семенчук В. Н. Разрешимые тотально локальные формации // Сиб. матем. ж. 1995. Т. 36. № 4. C. 869-872.

[11] Воробьев Н. Т. Локальные произведения классов Фиттинга // Весці АН БССР. Сер. фіз. -матэм. навук. 1991. №6. С. 28-32.

[12] Lockett F. P. The Fitting class $\mathfrak{F}^{*} / /$ Math. Z. 1974. V. 137. № 2. P. 131-136.

[13] Воробьев Н. Т. О радикальных классах конечных групп с условием Локетта // Матем. заметки. 1988. Т. 43. №2. С. 161-168.

[14] Ведерников В. А. О локальных формациях конечных групп // Матем. заметки. 1989. Т. 46. №6. C. $32-37$.

[15] Артамонов В.А., Салий В.Н, Скорняков Л.А., Шеврин Л.Н., Шульгейфер Е. Г. Общая алгебра. Т. 2. М.: Наука, 1991.

[16] Гретцер Г. Общая теория решеток. М.: Мир, 1982.

[17] Bryce R. A., Cossey J. Fitting formation of finite soluble groups // Math. Z. 1972. V. 127. № 3. P. 217-223.

Гомельский государственный университет им. $\Phi$. Скорины

Поступило

E-mail: vorobyov@gsu.unibel.by, skiba@gsu.unibel.by

22.04 .1999 